\title{
Supplementary Information for Ionic Effects on the Equilibrium Conformation of Catenated DNA Networks
}

\author{
Beatrice W. Soh*, Ahmed Khorshid*, Dana Al Sulaiman, and Patrick S. Doyle ${ }^{\dagger}$ \\ Department of Chemical Engineering, Massachusetts Institute of Technology, Cambridge, \\ Massachusetts 02139, USA.
}

\begin{abstract}
Algorithm for image processing
We used custom-developed MATLAB code to process the acquired images. For each recorded movie, we first manually select a region of interest (ROI) that encompasses the kinetoplast throughout the movie. Then, we perform a two-step background subtraction. The first step involves subtracting the average intensity of the pixels on the boundary of the ROI from the intensity of all pixels in the ROI. The second step looks at the remaining pixels with non-zero intensity values and removes bright, noisy spots that do not represent the kinetoplast. To determine whether a given pixel corresponds to the kinetoplast or noise, we calculate the average intensities of the eight pixels on the boundary of a three-by-three pixel box centered on the pixel of interest and the twelve pixels on the boundary of a four-by-four pixel box surrounding the pixel of interest. If either of the average intensities is less than $10 \sigma$, where $\sigma$ is the standard deviation of the intensity of the pixels on the boundary of the ROI, we determine the pixel to be noisy and set the intensity value to zero. We repeat the process for all images in the movie. The analysis code is available upon request.
\end{abstract}

${ }^{*}$ These authors contributed equally.

†pdoyle@mit.edu 


\section{Distributions of kinetoplast sizes}

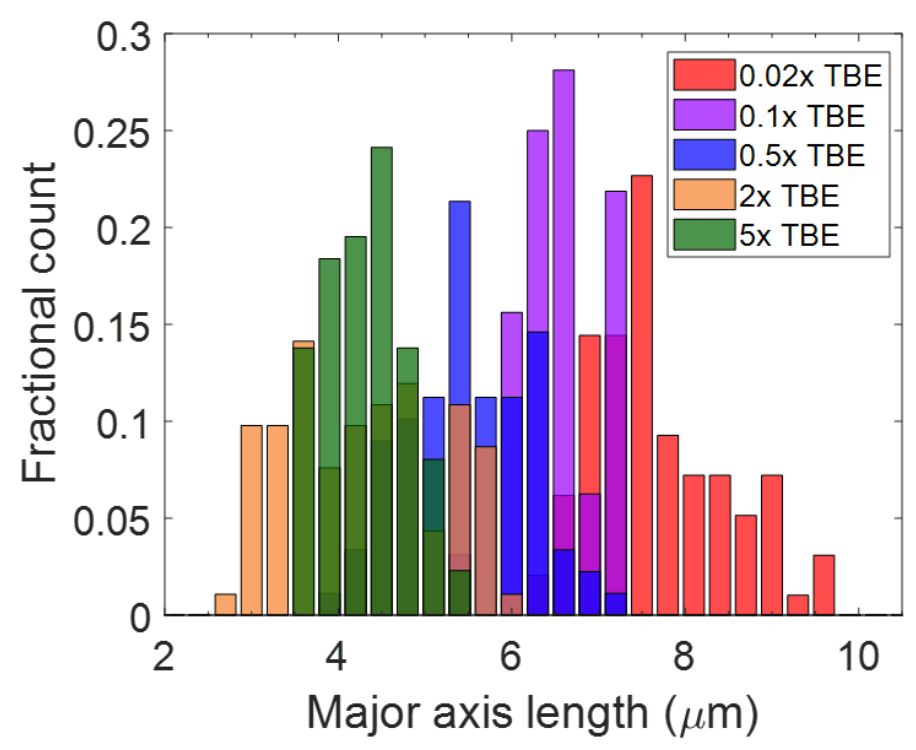

Figure S1: Histograms of major axis lengths for all ionic strengths investigated.

Fig. S1 shows the distributions of kinetoplast sizes across all ionic strengths studied in this work. The number of kinetoplasts in each ensemble are 97 (0.02x TBE), 32 (0.1x TBE), 89 (0.5x TBE), 92 (2x TBE) and 87 (5x TBE).

\section{Excluded volume parameter for $2 \mathrm{D}$ polymer model}

It is well known that for linear polymers with Kuhn length $b$ much longer than width $w$, the mean-field excluded volume per Kuhn monomer $b^{2} w$ is much larger than the occupied volume $b w^{2}{ }^{1}$ The goal of this section is to calculate the effective excluded volume parameter for a $2 \mathrm{D}$ polymer network. Fig. S2 shows the 2D polymer model used to develop the excluded volume term in the Flory type argument presented in the main text. To develop a tractable scaling theory, we have simplified the complex geometry of the kinetoplast to a series of linear polymer chains of width $w$, with $n_{c}$ segments of Kuhn length $b$ connected at fixed vertices. For simplicity, we show the calculation for an open square lattice. Other open lattice geometries would simply introduce a different dimensionless numerical prefactor in our final calculation, which would then be neglected in a scaling argument.

Our calculation closely follows the lucid derivation for linear polymers in the text by Rubinstein and Colby. ${ }^{1}$ We hence start with a simplified representation of the kinetoplast as spherical monomers of diameter $w$ on an open square lattice, which can be coarse-grained into cylindrical monomers of diameter $w$ and Kuhn 

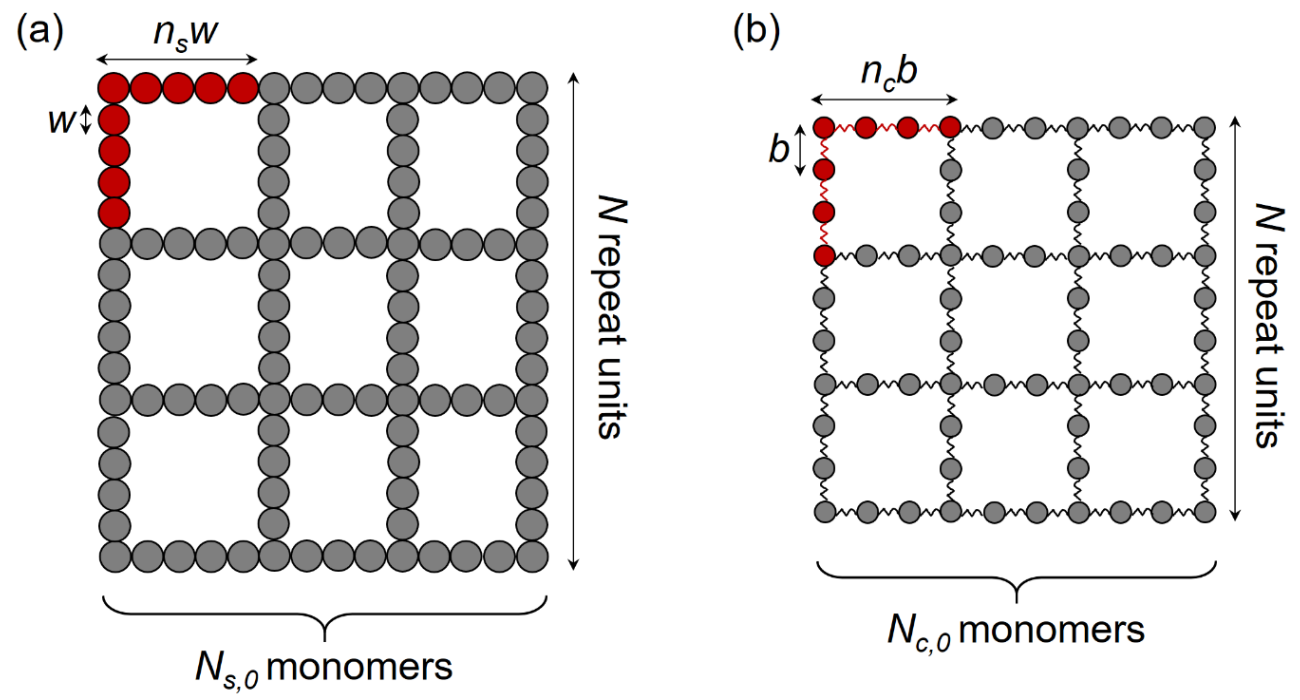

Figure S2: (a) Open lattice representation of a 2D polymer with spherical monomers of diameter $w$. (b) Coarsegrained representation of a 2D polymer with cylindrical monomers of Kuhn length $b$ and diameter $w$, and $n$ monomers between fixed vertices. The repeat units are colored in red.

length $b$ on a square lattice. The repeat unit of the polymer, colored red in Fig. S2, consists of $n_{s}$ spherical monomers of diameter $w$ along each edge, or $n_{c}$ cylindrical monomers of Kuhn length $b$ along each edge. From the mapping of repeat units between the two representations, it follows that

$$
n_{s} w=n_{c} b .
$$

Excluded volume describes the interactions between monomers in solution. Considering only two-body interactions, the interaction part of the free energy for a polymer in bulk is described by ${ }^{1}$

$$
\frac{F_{\text {int }}}{k_{B} T} \sim \frac{v N_{\text {tot }}^{2}}{R^{3}}
$$

where $k_{B} T$ is the thermal energy, $v$ is the mean-field approximated excluded volume parameter, $N_{\text {tot }}$ is the total number of monomers in the system and $R$ is the size of the confined polymer. For spherical monomers of diameter $w$, we have $v \sim w^{3}$. The interaction energy must not change regardless of the choice of spherical monomers (Fig. S2a) or cylindrical monomers (Fig. S2b). Hence, we require for the polymer

$$
v_{s} N_{\mathrm{tot}, \mathrm{s}}^{2}=v_{c} N_{\mathrm{tot}, \mathrm{c}}^{2}
$$

where $v_{s}$ and $v_{c}$ are the excluded volume parameters for spherical and cylindrical monomers respectively, and $N_{\text {tot,s }}$ and $N_{\text {tot,c }}$ are the total number of spherical and cylindrical monomers respectively.

First, we look at the total number of spherical monomers in the system. As seen from Fig. S2, the total 
number of repeat units is $N^{2}$ and the number of monomers per repeat unit is $2 n_{s}-1$. Therefore, the total number of spherical monomers is obtained as

$$
N_{\text {tot, } \mathrm{s}}=N^{2}\left(2 n_{s}-1\right)
$$

Next, we consider the total number of cylindrical monomers $N_{\text {tot,c }}$ in the system. The total number of repeat units is $N^{2}$ and the total number of monomers per repeat unit is $2 n_{c}$. Hence, the total number of cylindrical monomers is given by

$$
N_{\text {tot,c }}=2 N^{2} n_{c}
$$

Substituting Eqs. 4 and 5 into Eq. 3, we have

$$
v_{s} N^{4}\left(2 n_{s}-1\right)^{2}=4 v_{c} N^{4} n_{c}^{2}
$$

or,

$$
v_{s}\left(4 n_{s}^{2}-4 n_{s}+1\right)=4 v_{c} n_{c}^{2}
$$

Keeping only the leading order term in Eq. 7 and taking into account $v_{s} \sim w^{3}$, we obtain

$$
v_{c} \sim w^{3}\left(\frac{n_{s}}{n_{c}}\right)^{2}
$$

Substituting in Eq. 1, we arrive at

$$
v_{c} \sim w^{3}\left(\frac{b}{w}\right)^{2} \sim b^{2} w .
$$

It is interesting to note that the excluded volume parameter $v_{c}$ for our $2 \mathrm{D}$ polymer model is the same as that for $1 \mathrm{D}$ polymers. ${ }^{1}$ 


\section{Scaling argument using a blob model}

We can use a blob model to develop the same scaling argument as that obtained from a Flory type approach in the main text. The size of a slit confined polymer $R_{\|}$is given by

$$
R_{\|} \sim h n_{\mathrm{blob}}^{1 / 2}
$$

where $h$ is the channel height equal to the blob size and $n_{\text {blob }}$ is the number of blobs. Inside a blob, we have Flory scaling in the bulk

$$
h \sim b^{2 / 5} v^{1 / 5} n_{\mathrm{mer}}^{4 / 5}
$$

where $b$ is the Kuhn length, $v$ is the excluded volume parameter and $n_{\text {mer }}$ is the number of monomers in a blob. We solve for the number of monomers in a blob

$$
n_{\text {mer }} \sim h^{5 / 4} b^{-1 / 2} v^{-1 / 4} .
$$

The mass of monomers in a blob $m_{\mathrm{mer}}$ is thus

$$
m_{\mathrm{mer}} \sim n_{\mathrm{mer}}^{2} \sim h^{5 / 2} b^{-1} v^{-1 / 2}
$$

We obtain the total number of blobs $n_{\text {blob }}$

$$
n_{\text {blob }} \sim \frac{M}{m_{\text {mer }}} \sim \frac{N^{2}}{m_{\text {mer }}} \sim N^{2} h^{-5 / 2} b v^{1 / 2}
$$

where $M$ is the total mass of the polymer and $N$ is the total number of monomers in a given direction. Substituting Eq. (14) into Eq. (10), we have

$$
R_{\|} \sim h n_{\mathrm{blob}}^{1 / 2} \sim h^{-1 / 4} N b^{1 / 2} v^{1 / 4}
$$

Taking into account $N=L / b$ and $v \sim b^{2} w$, where $L$ is the linear size of the polymer and $w$ is the effective width, we arrive at

$$
R_{\|} \sim \frac{w^{1 / 4} L}{h^{1 / 4}}
$$

as was obtained from the Flory type approach presented in the main text. 


\section{Ratio of polymer charge concentration to salt concentration}

To estimate the polymer charge concentration, we assume the kinetoplast to have dimensions of $5 \mu \mathrm{m}$ by 5 $\mu \mathrm{m}$ by $3 \mu \mathrm{m}$, resulting in a volume of $75 \mu \mathrm{m}^{3}$, or $7.5 \times 10^{-14} \mathrm{~L}$. Taking into account 5000 minicircles of 2.5 kbp each and 25 maxicircles of $40 \mathrm{kbp}$ each, a single kinetoplast has $1.35 \times 10^{7}$ base pairs. Since every base pair carries two negative charges from the phosphate groups, each kinetoplast has a total of $2.7 \times 10^{7}$ charges along the polymer backbone. This leads to a charge density of $6.0 \times 10^{-4} \mathrm{M}$ for each kinetoplast. The lowest ionic strength used in the experiments is $4.74 \times 10^{-3} \mathrm{M}$ (see Table 1 in main text). Even without accounting for the reduced effective charge on the polymer due to counterion condensation, the salt concentrations used in the experiments are at least an order of magnitude greater than the kinetoplast charge concentration. Hence, the interactions between monomers are predominantly short-range and can be viewed as excluded volume interactions. ${ }^{2}$

\section{Principal component analysis for kinetoplast shape}

(a)

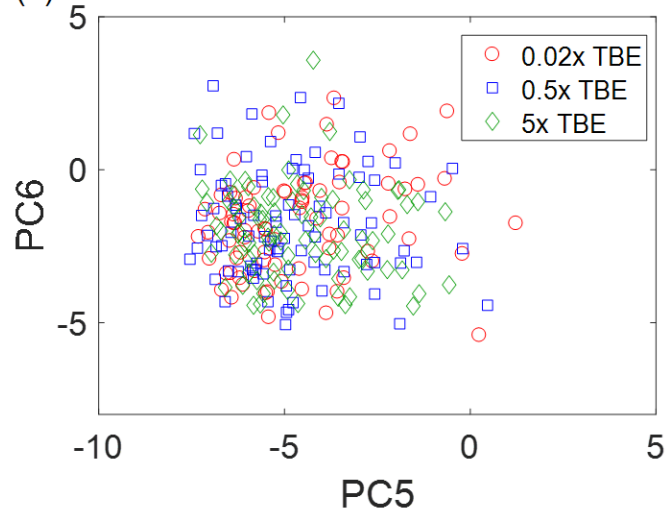

(b)

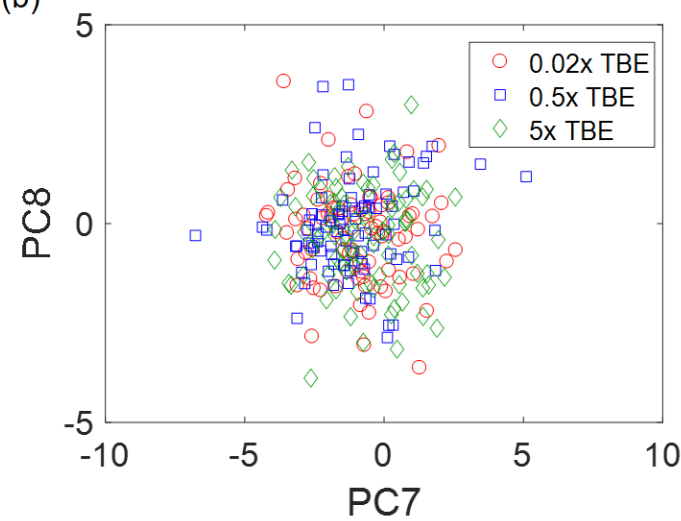

Figure S3: Scatter plots of the (a) fifth and sixth PC amplitudes, and (b) seventh and eighth PC ampitudes for ensembles of kinetoplasts in 0.02x TBE, 0.5x TBE and 5x TBE.

Fig. S3 shows the locations of each kinetoplast outline in the PC5-PC6 and PC7-PC8 space. We note that there is no obvious shift in distribution of locations in the higher PC space with a change in ionic strength, further supporting the notion that the shapes of kinetoplasts are not noticeably affected by ionic strength.

In the main text, we applied PCA to a combined ensemble of kinetoplasts in $0.02 \mathrm{x}$ TBE, $0.5 \mathrm{x}$ TBE and 5x TBE. Alternatively, we can apply PCA to each ensemble at a different ionic strength and consider the PCs identified for each population of kinetoplasts. Fig. S4 shows images of the first eight PCs for each ensemble of kinetoplasts in 0.02x TBE, 0.5x TBE and 5x TBE, along with plots of the first twenty PCs. 
(a)

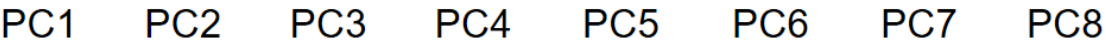

$0.02 \times$ TBE<smiles>C1CCCCC1</smiles><smiles>C1CCCCC1</smiles><smiles>C1CC2CCC1C2</smiles><smiles>C1CC2CCC1C2</smiles><smiles>C1CCCCC1</smiles><smiles>C1CCCCC1</smiles><smiles>C1CCCCC1</smiles>

$0.5 \times \mathrm{TBE}$<smiles>C1CCCCCC1</smiles><smiles>C1CCCCC1</smiles><smiles>C1CCCC1</smiles><smiles>C1CCCC1</smiles><smiles>C1CCCCC1</smiles><smiles>C1CCCCC1</smiles><smiles>C1CCCCC1</smiles><smiles>C1CCCCC1</smiles>
$5 \times \mathrm{TBE}$<smiles>C1CCCCC1</smiles>
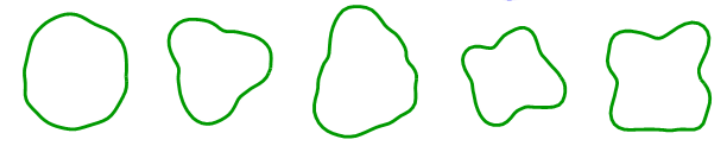

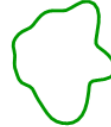

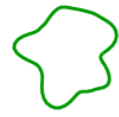

(b)

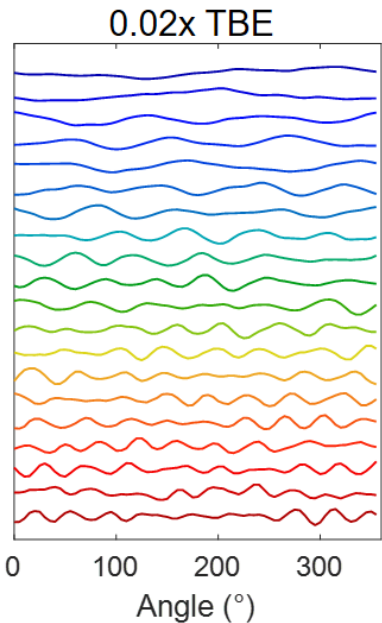

$0.5 \times \mathrm{TBE}$

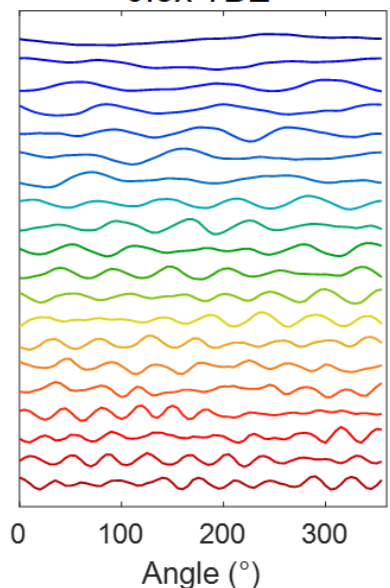

$5 x$ TBE

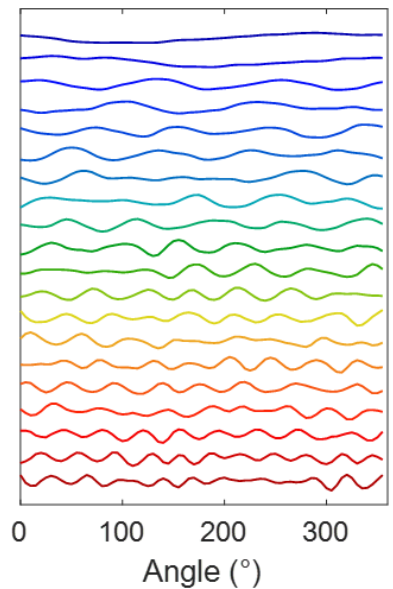

Figure S4: Images of the first eight principal components (PCs) for ensembles of kinetoplasts in $0.02 \mathrm{x}$ TBE, 0.5x TBE and 5x TBE. (b) Plots of the first twenty PCs for ensembles of kinetoplasts in 0.02x TBE, 0.5x TBE and 5x TBE on shifted y-axes.

Qualitatively, we do not observe any discernible differences in the first eight PCs, with the images for the PCs of different ensembles looking approximately to be rotations relative to each other, or in the plots of the first twenty PCs, with the evolution in the number of peaks and troughs for each PC appearing to be similar. The result of analyzing each ensemble at a different ionic strength using PCA is consistent with that obtained from analyzing the combined ensemble, showing that the shape of kinetoplasts does not exhibit a strong dependence on ionic strength. 


\section{References}

[1] M. Rubinstein and R. H. Colby. Polymer Physics. Oxford University Press, 2003.

[2] A.V. Dobrynin and M. Rubinstein. Theory of Polyelectrolytes in Solutions and at Surfaces. Progress in Polymer Science, 30(11):1047-1118, 2005. 